\title{
Enhanced properties of UPE/ESOA partially bio-nanocomposites reinforced with chitosan functionalized graphene nanoplatelets: an innovative approach
}

\author{
SHIVKUMARI PANDA*, DIBAKAR BEHERA, PRASANT RATH and TAPAN KUMAR BASTIA \\ School of Applied Sciences (Chemistry), KIIT University, Bhubaneswar, Odisha 751024, India \\ *Author for correspondence (Shivkumaripanda@gmail.com)
}

MS received 17 July 2017; accepted 6 December 2017; published online 25 July 2018

\begin{abstract}
The current study deals with the successful development of chitosan-functionalized graphene nanoplatelets (CS/GNPs) and their dispersion in the unsaturated polyester (UPE)/epoxidized soybean oil acrylate (ESOA) (80:20 w/w) blend system in different compositions of $0.3,0.5$ and $0.7 \mathrm{wt} \%$. The resulting nanocomposite mixture was achieved by simple sonication method and pressed into a mould for fabrication of nanocomposite. The whole functionalization and nanocomposite preparation procedure were successfully tracked by FTIR, SEM and TEM. Nanocomposite with $0.5 \mathrm{wt} \%$ of CS/GNPs nanofiller has demonstrated it as the better candidate due to its optimum properties. Again for better comparison, a nanocomposite with $0.5 \mathrm{wt} \%$ raw GNPs was also fabricated and its properties were studied in detail. This relative study reported lower mechanical, dynamic mechanical, thermal and electrical conductivity values for $0.5 \mathrm{wt} \%$ raw GNPs than the corresponding CS/GNPs nanofiller-filled nanocomposites. Nanocomposite with $0.5 \mathrm{wt} \%$ CS/GNPs showed dramatic enhancement in mechanical, dynamic mechanical, thermal and electrical properties as well as reduced corrosion and swelling performance owing to the homogeneous distribution of nanofiller in the blend. Again, the nanocomposite showed the highest thermal and electrical conductivities with the best dielectric strength. Thus, the prepared nanocomposites with optimum nanofiller content might serve as partially biodegradable nanomaterial for applications in nanotechnology engineering, thermal applications, such as circuit boards and electrical applications, such as electronic packaging components, electromechanical devices and electric energy storage devices. This nanocomposite can also find their applications in different corrosive- and solvent-based environments.
\end{abstract}

Keywords. Nanocomposites; reinforcements; mechanical properties; thermal properties.

\section{Introduction}

Recently, the mainstream of the scientific community has devoted much attention on nanotechnology and nanomaterials. These unique novel nanomaterials exhibit some superior characteristics, as compared to other typical macroand micro-sized materials. There were growing interests in incorporating carbon-based nanofillers, such as carbon black, expanded graphite and carbon nanotube (CNT) in the preparation of polymeric nanocomposites [1,2]. Currently, graphene nanoplatelets (GNPs), one of the stiffest known 2D honeycomb-type carbon materials have achieved the extensive attention of the modern scenario owing to its inherently high mechanical strength with good electrical and thermal conductivities. So, GNPs are regarded as promising carbon nanofiller for nanocomposite due to their good balance of properties and cost [3-5]. However, due to the thermodynamically unstable large-specific surface area, strong Vander Waals bond and $\pi-\pi$ stacking forces between layers, GNPs usually tend to agglomerate into the matrix $[6,7]$. A number of methods were discovered to enhance the dispersability of GNPs into the polymer matrix. The incorporation of well-dispersed graphene into polymers can remarkably improve the properties of the polymer host materials [8]. Various covalent and non-covalent chemical functionalization methods of GNPs with their introduction into various polymers for preparing nanocomposite were already published by various authors [9-12]. Here, we have conducted non-covalent functionalization of GNPs with a biomaterial to expand and optimize its utilization. Chitosan (CS) is a very interesting, abundant, non-toxic, biocompatible, biological Ndeacetylated derivative of chitin $[13,14]$. It is easily obtained from the shell wastes of crustaceans, shrimps and crabs. Due to better chemical and physical properties, chitosan was given credit as a functional biopolymer and nanobiotechnologybased applications [15]. Chitosan-grafted GNPs (CS/GNPs) may offer a novel approach to the design of high performance nanocomposite with better applicability. Here, attempts were made to achieve stable and fine-dispersed GNPs in a suitable dispersing medium of ethanol. Then, these well-dispersed GNPs are functionalized with chitosan.

Blending of polymers is promising as superior polymeric materials. It will also give an innovative and improved combination of two different types of unique properties. Generally, 
unsaturated polyester (UPE), a thermosetting resin is used in many fields due to its brittleness. But they possess poor damage tolerance, high viscosity, low mechanical strength and stiffness relating to other engineering materials such as metals [16]. Thus, UPE was blended with epoxidized soybean oil acrylate (ESOA) to recuperate its performance. ESOA is a vegetable oil-based plasticizer with biodegradable, intoxic, inexpensive, easily available, biocompatible with good lubricity, high viscosity index, solvency for lubricant additive and easily miscible nature of the polymer.

In this work, the optimal 80:20 (w/w) UPE-ESOA blend composition, which is already reported in our previous work [17] was taken as the blended matrix due to its comparatively better properties. To study the reinforcing effect of CS/GNPs, primarily $0.5 \mathrm{wt} \%$ of non-functionalized GNPs $(0.5 \mathrm{r}(\mathrm{G}))$ was referred. Then, nanocomposites reinforced with $0.3,0.5$ and $0.7 \mathrm{wt} \% \mathrm{CS} / \mathrm{GNPs}$ were fabricated. Now, the mechanical, dynamic mechanical, thermal, electrical, corrosion and swelling properties were studied with respect to the GNPs content. All the described properties were already reported for UPE/ESOA blend [17]. Here, the nanocomposite showed steady enhancement in all the above properties than the neat blend with minor corrosion and swelling properties. It is only due to the addition of CS/GNPs into the blend, optimum improvement was observed in CS/GNPs with $0.5 \mathrm{wt} \%$ nanocomposite. In the relative study of nanocomposites with varying contents of raw and functionalized nanofillers, it was quite interesting to note that the nanocomposite with $0.5 \mathrm{wt} \% \mathrm{CS} / \mathrm{GNPs}$ kept its ascendancy over other nanocomposites with least corrosion and swelling. This may be attributed to the better dispersion of hydrophobic GNPs in ethanol medium and successful non-covalent functionalization of CS/GNPs that induced the uniformity of mixing in the blend at $0.5 \mathrm{wt} \%$ concentration.

\section{Experimental}

\subsection{Materials}

GNPs of $>98 \%$ carbon content, diameter of $5-7 \mathrm{~nm}$, length of $5 \mu \mathrm{m}$ and density of $\sim 2.28 \mathrm{~g} \mathrm{~cm}^{-3}$ were supplied by Sigma Aldrich, USA. Chitosan (CS-80\% deacetylated, $M_{\mathrm{w}}=100 \mathrm{KDa}$ ), methyl ethyl ketone peroxide (MEKP, catalyst), cobalt naphthanate (accelerator) and ethanol were purchased from Sigma Aldrich. Isophthalic polyester (unsaturated polyester, UPE) was used as the resin (supplied by Vasavi Bala Resins, VBR-4301 and viscosity 0.3 Pa.s). The ESOA was synthesized by using the reported method [17] in our laboratory from ESO. Similarly, the preparation of UPE-ESOA blend (80:20) was also reported [17]. All required reagents were analytical grade and used without any purification.

\subsection{Methods}

2.2a Chitosan functionalization of GNPs: At first, $2 \mathrm{~g}$ of GNPs were dispersed in $50 \mathrm{ml}$ of water/ethanol (1:3 (v/v)) solution. Then, the suspension was sonicated at $50^{\circ} \mathrm{C}$ at a frequency of $40 \mathrm{kHz}$ for $9 \mathrm{~h}$. Prior to the CS usage, it was completely deacetylated by adding $80 \%$ deacetylated CS to $50 \% \mathrm{NaOH}$ solution and stirring for $8 \mathrm{~h}$ at $90^{\circ} \mathrm{C}$ under nitrogen atmosphere. The product was filtered and washed with methanol and dried repetitively thrice. About $4 \mathrm{~g}$ of this dried chitosan was treated with $0.5 \mathrm{M} \mathrm{HCl}$ solution. Finally, the GNPs-ethanol suspension was gradually added to the chitosan-acid solution and the mixture was stirred at $70^{\circ} \mathrm{C}$ for $4 \mathrm{~h}$ to yield a uniform black-coloured solution. Then, the mixture was filtered through a $0.2 \mu \mathrm{m}$ microporous poly(ether sulfone) membrane, washed with distilled water to take away the adsorbed and uncross-linked CS. Now, the product was dried in vacuum oven at $80^{\circ} \mathrm{C}$ for $48 \mathrm{~h}$. The end product was chitosan-functionalized GNPs and the complete scheme of preparation is presented in figure 1 .

2.2b Fabrication of nanocomposites: Initially, the CSfunctionalized GNPs apart from the curing agent, to put off early curing reaction, were added carefully in varied weight ratios $0.3,0.5$ and $0.7 \mathrm{wt} \%$ with the UPE/ESOA blend followed by sonication in a high intensity ultra-sonicator at about $60^{\circ} \mathrm{C}$ for $1.5 \mathrm{~h}$ with mechanical stirring. Exterior cooling system was provided by submerging the beaker in an ice bath to circumvent the temperature rise during the sonication procedure, followed by stirring overnight to gain a consistent and steady suspension. Then, $3 \% \mathrm{MEKP}(\mathrm{w} / \mathrm{w}$ ) catalyst and $0.5 \%$ cobalt naphthanate (w/w) accelerator were added and mixed further by sonicating for $30 \mathrm{~min}$. Then, gel coat with $2 \%$ (w/w) DCP was homogeneously brushed into a mould and the prepared nanocomposite mixture was poured one at a time for the fabrication. The mould was subjected to hot-press at $120^{\circ} \mathrm{C}$ for $2 \mathrm{~h}$ with a pressure of 5 tons. Similar to the above method, the nanocomposite containing $0.5 \mathrm{r}(\mathrm{G})$ were also prepared.

\section{Instruments and measurements}

\section{$3.1 \quad$ FTIR}

Fourier transform infrared spectroscopy (FTIR) spectra of CS, GNPs, CS-non-covalent functionalized GNPs (CS/GNPs) and the CS/GNPs nanocomposites were collected using Thermo-Nicolate Model 400 instrument equipped with a controlled temperature cell (Model HT-32 heated demountable cell used with an Omega 9000-A temperature controller).

\subsection{Raman spectroscopy}

Raman spectroscopy was used to record the peaks to determine the layer of pristine GNPs, CS-functionalized GNPs and CS/GNPs-reinforced nanocomposites on a confocal 


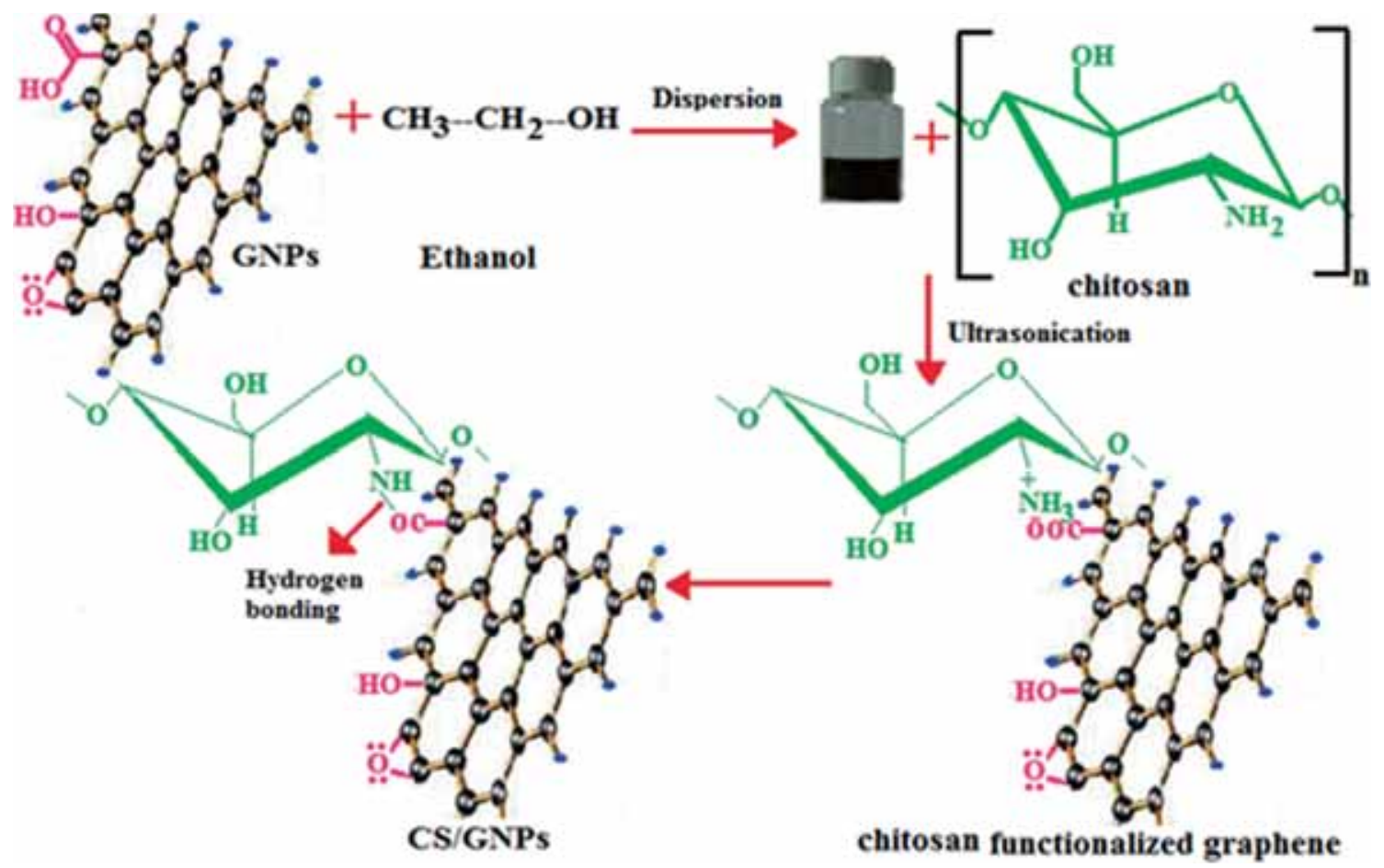

Figure 1. Schematic diagram for functionalization mechanism of GNPs.

micro-Raman microscope (Renishaw in via Reflex) with excitation laser of $515 \mathrm{~nm}$ wavelength and $50 \times$ objective lens was used to evaluate the layer of GNPs and its presence in nanocomposites.

\subsection{SEM}

Scanning electron microscopy (SEM) was utilized to study qualitatively the external morphology of CS, GNPS, GNPs CS-non-covalent functionalized GNPs (CS/GNPs) and the CS/GNPs nanocomposites. The samples were gold-coated and examined by using a Philips 420T SEM with a secondary electron detector, operating at $20 \mathrm{kV}$ in the SEM mode.

\subsection{TEM}

Transmission electron microscope (TEM) was performed in JEM-200FX. Thin sections ( $90 \mathrm{~nm}$ ) of the cured samples were obtained by microtome with diamond knife for TEM analysis. The filament voltage was kept $200 \mathrm{kV}$ to construct a clear field image of the nanostructure.

\subsection{Mechanical testing}

Tensile properties were evaluated by using the universal testing machine (model 3369) in accordance with ASTM D790-03 standard. IZOD impact strength was measured in an impact tester as per D256-05. Hardness was measured by using a durometer (hardness Shore A) according to
ASTM-D2240 standard by a pointed needle, which was directly applied on to the surface of the samples. All the measurements were taken as an average of four samples, because the properties for UPE/ESOA blend were previously studied [17].

\section{$3.6 D M A$}

For dynamic mechanical analysis (DMA), four test specimens $(56 \times 13 \times 3 \mathrm{~mm})$ were cut from the centre section of an ASTM type I tensile bar. The dynamic mechanical properties, like storage modulus and damping coefficient $(\tan \delta)$ were evaluated with a DMA tester (model Q800).

\subsection{TGA}

Thermogravimetric analysis (TGA) was conducted on a Perkin-Elmer Series 7 thermal analyzer with an air purge in a dry nitrogen atmosphere at a heating rate of $10^{\circ} \mathrm{C} \mathrm{min}{ }^{-1}$. The weight loss of the sample was measured as a function of temperature.

\subsection{Thermal conductivity measurement}

This was studied with thermal conductivity instrument (TCI) -2022 SX211 as per ASTM E 1530 standard below $10^{-5}$ torr vacuity environment. The samples were cut into $(50 \times 10 \mathrm{~mm})$ for this study. The thermal conductivity was calculated in the temperature range of -50 to $150^{\circ} \mathrm{C}$. Here, the measurements 
were taken for five samples, because the properties for UPE/ESOA blend are studied here.

\subsection{Electrical properties measurement}

Dielectric strength was measured according to IEC-60243-1 (ASTM D 149) standard at $250 \mathrm{~V}$ and $50 \mathrm{~Hz}$. Volume and surface resistance of the specimens were studied according to ASTM D257 by using Keithley 6517 A model 8009 resistivity test fixture.

\subsection{Corrosion testing}

Initially, the edges of the samples were sealed with UPE resin and their initial weights were taken in dry condition in an electronic balance with the accuracy of $1 \times 10^{-5} \mathrm{mg}$ and model AY 220. The corrosion of the samples $\left(12 \times 10 \times 3 \mathrm{~mm}^{3}\right)$ on exposure to salt water were calculated by dipping them in $10 \mathrm{wt} \% \mathrm{NaCl}$ concentration, $\mathrm{pH}$ value of 6.5 at room temperature by using ASTM B117 standard. After a gap of 1, 2 and 3 days of testing, each time, the samples were thoroughly rinsed in deionized water and dried in an oven for $12 \mathrm{~h}$ and final weights were taken.

\subsection{Swelling experiments}

This experiment was performed in water, dichloroethylene $\left(\mathrm{CH}_{2} \mathrm{Cl}_{2}\right)$ and methyl ethyl ketone (MEK) at room temperature. Five nanocomposite specimens $(3 \times 3 \times 5 \mathrm{~mm})$ were kept immersed in each of the above liquids for about 30 days. The swelling equilibrium was determined when the weight of the swollen samples were maintained constant. The initial weight of the specimens was taken in swollen state. Now, the swollen specimens were washed with fresh solvent and desiccated under vacuum at $150^{\circ} \mathrm{C}$ for three days until constant weight was achieved.

\section{Results and discussion}

\subsection{Proposed reaction mechanism of CS-functionalized GNPs and the nanocomposites}

The whole functionalization mechanism of GNPs with schematic diagram is conceptualized in figure 1 and was confirmed by the FTIR analysis shown in figure 3. GNPs containing various reactive sites were pre-treated with ethanol. Then, during sonication with chitosan, the amine groups of chitosan might get attached with the carboxylic acid group of the nanofiller through van der Waals force of attraction. Now, the sticked-out hydrophilic moieties of the chitosan affixed on the GNP surfaces, encourage repulsion between the GNPs. As a result, this phenomenon may produce better dispersion of the GNPs due to the retention effect of perfectly covered GNPs with chitosan. Figure 2 shows the possible detailed reaction mechanism for the formation of CS/GNPs-reinforced UPE/ESOA nanocomposite. This figure suggests the chance of occurrence of hydrogen bonding

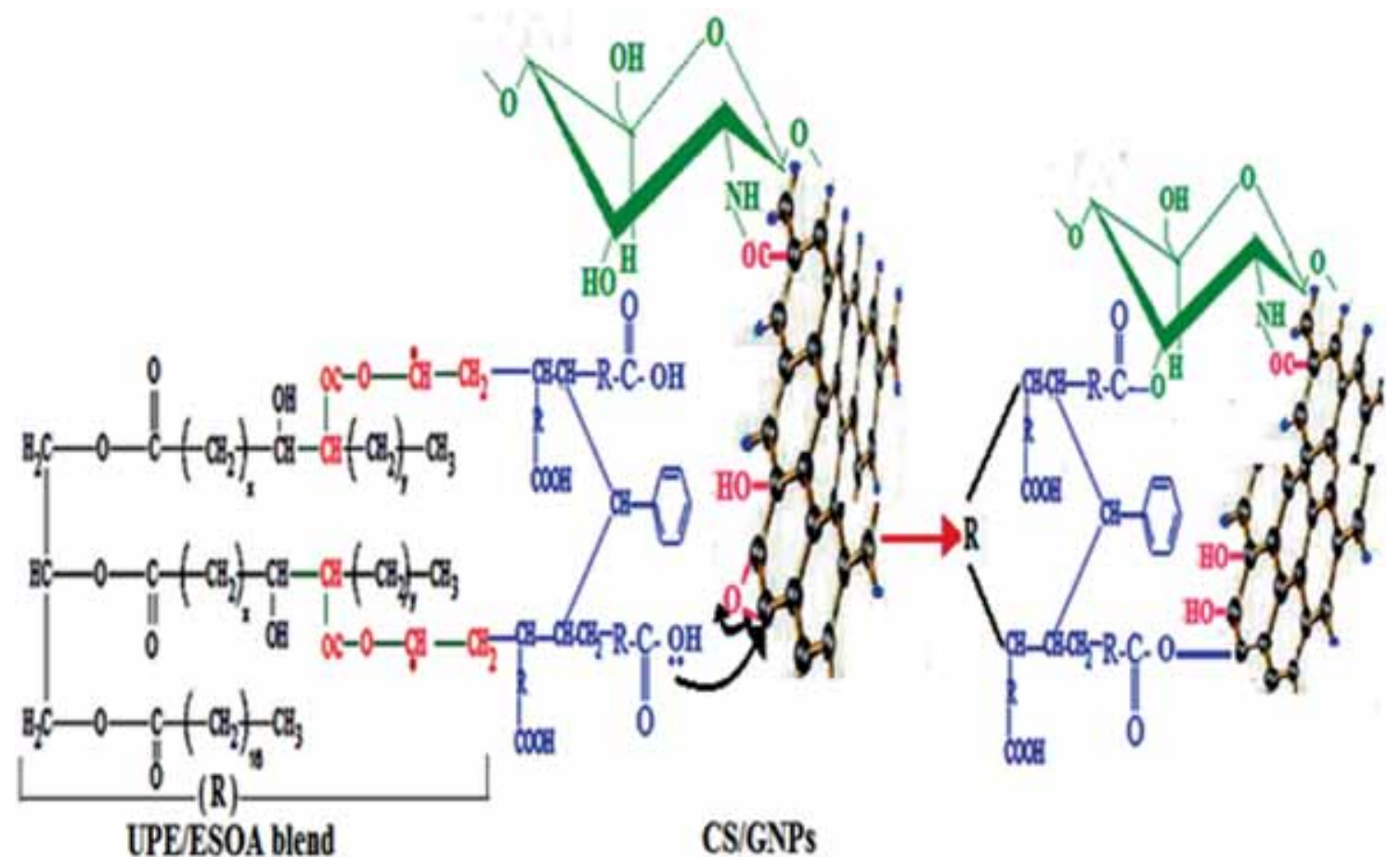

Figure 2. Possible reaction mechanism of CS/GNPs-reinforced UPE/ESOA nanocomposite. 
between $-\mathrm{OH}$ group of chitosan and $-\mathrm{COOH}$ group of UPE/ESOA blend. Then, an ester linkage was formed with the blend in the nanocomposite by removing water as a side product.

Again, amide bond is formed between the carboxylic acid group of GNPs and amine group of chitosan. In addition to this, a typical reaction occurred between epoxy group of GNPs and carboxyl group of the blend results in a repeating unit, containing both an ester and hydroxyl functional groups. The proper formation of nanocomposite is further confirmed by FTIR analysis given in figure 3.

\subsection{FTIR analysis}

FTIR spectra of (a) CS- (b) GNPs- (c) CS/GNPs- and (d) CS/GNPs-reinforced UPE/ESOA nanocomposite are shown in figure 3. Figure $3 \mathrm{a}$ shows peaks at 3429 and $3352 \mathrm{~cm}^{-1}$ corresponding to $-\mathrm{OH}_{\text {str }}$ and $\mathrm{NH}_{\text {str }}$ vibrations, respectively. $\mathrm{NH}_{\text {bend }}$ and $\mathrm{NH}_{\text {def }}$ vibrations of $\mathrm{NH}_{2}$ are observed at 1655 and $1425 \mathrm{~cm}^{-1}$, respectively. A peak at $1085 \mathrm{~cm}^{-1}$ attributed to the $\mathrm{CO}_{\text {str }}$ vibration of $\mathrm{COH}$, revealing that all the functional moieties were originally present in chitosan. As shown in figure $3 \mathrm{~b}$, major bands appeared in the FTIR spectra of

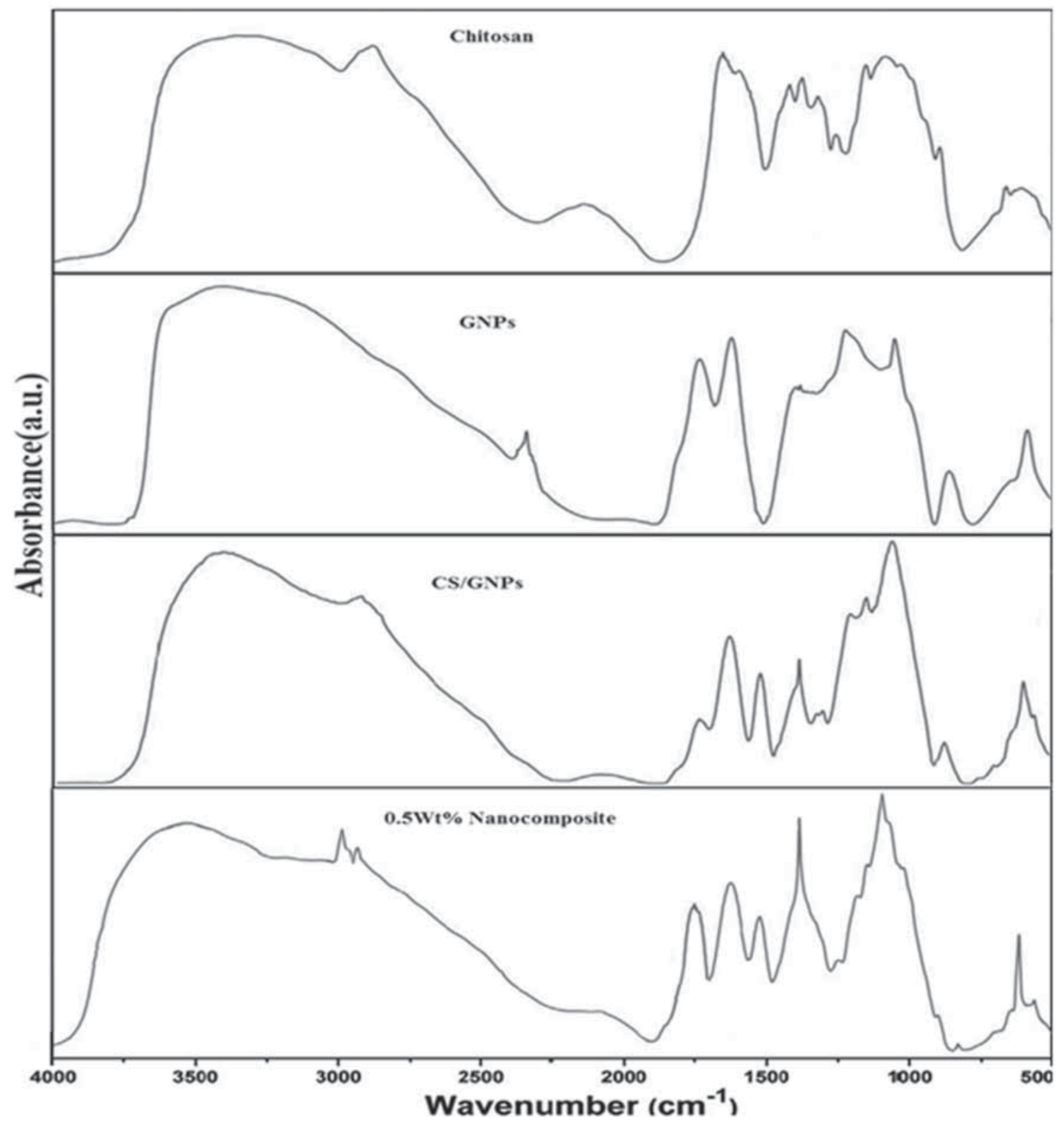

Figure 3. FTIR of deacetylated chitosan, neat GNPs, CS/GNPs and the $0.5 \mathrm{wt} \%$ CS/GNPs nanocomposite. 
pristine GNPs, signify the existence of functional groups like carbonyl peak at $1740 \mathrm{~cm}^{-1}$, hydroxyl peak at $3452 \mathrm{~cm}^{-1}$ and carboxyl band at $1670 \mathrm{~cm}^{-1}$, which are essential for making reaction with UPE/ESOA blend. Figure $3 c$ depicts the spectra of the CS/GNPs functionalization peak. Here, the peaks at 1085 and $3452 \mathrm{~cm}^{-1}$ have greater intensities than that of normal peaks. It may be due to the successful reaction of hydroxyl group of GNPs with chitosan. Again, the peak of carboxylic group at $1670 \mathrm{~cm}^{-1}$ was shifted to a lower frequency, confirming that the carboxylic groups reacted with chitosan during the preparation of CS/GNPs functionalization as given in figure 1. Again, after surface decoration of $\mathrm{CS}$, a new stake-shaped absorbance peak at $1652 \mathrm{~cm}^{-1}$ was observed indicating the formation of $\mathrm{NH}-\mathrm{CO}$ (amide) group. This shows that chitosan has $-\mathrm{NH}_{2}$ groups in its molecular chains that react with $-\mathrm{COOH}$ functional group of GNPs. As seen in figure 2 , the similar peak at $1165 \mathrm{~cm}^{-1}$ attributed to the minor shift of $\mathrm{C}-\mathrm{O}-\mathrm{C}_{\text {ester }}$ of CS/GNPs with the blend. The peaks appear at 1655 and $1760 \mathrm{~cm}^{-1}$ correspond to the secondary amide group of the nanocomposite and $\mathrm{C}-\mathrm{O}_{\text {ester }}$ group of the blend. This provides a good indication of construction of the nanocomposite with the possibility of reaction between the nanofiller and the blend by the successful attachment of CS-functionalized GNPs to the blend.

\subsection{Raman spectroscopy}

The Raman spectra of raw GNPs-, CS/GNPs- and CS/GNPsreinforced UPE/ESOA nanocomposites with varying amounts of nanofiller concentration are given in figure 4. In figure 4a, the sharp peaks are appeared at $1587.63 \mathrm{~cm}^{-1}$ corresponding to G-band. The shape and position of the G-band are due to the in-plane stretching motion between $\mathrm{SP}_{2}$ carbon atoms and depend on the number of layers of the GNPs. Another important finger print of GNPs with strong, single

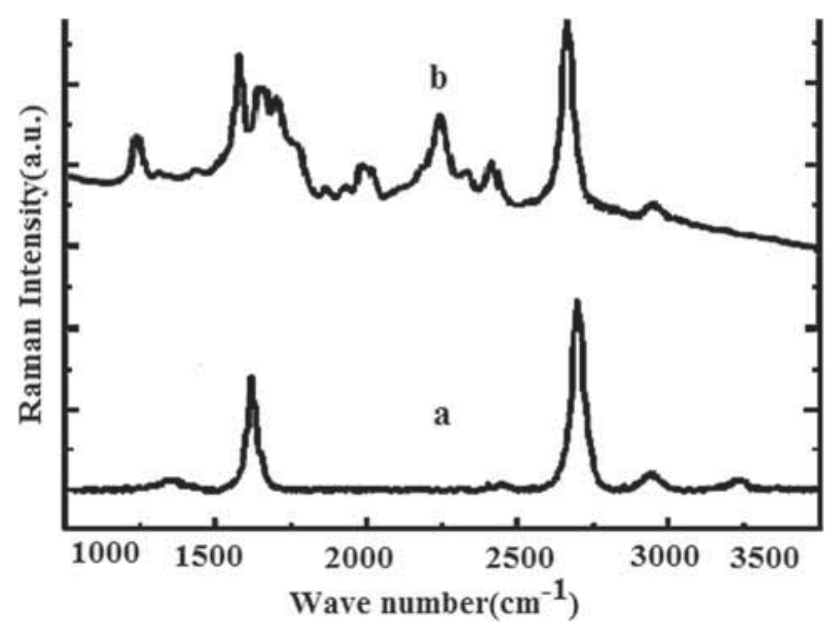

Figure 4. Raman spectra of (a) GNPs- and (b) chitosanfunctionalized GNPs chitosan, neat GNPs, CS/GNPs and the $0.5 \mathrm{wt} \% \mathrm{CS} / \mathrm{GNPs}$ nanocomposite. and symmetric peak at $2686 \mathrm{~cm}^{-1}$ is associated with the $2 \mathrm{D}$ band. Here, D-band is weak. Now, the detailed characteristics regarding the number of atomic layers present in the GNPs can be calculated from this Raman spectroscopy study by using the following equation.

$$
W_{\mathrm{G}}=1581.6+\frac{11}{1+n^{1.6}}
$$

where $W_{\mathrm{G}}$ is the wave number for band position and $n$ the number of layers of the GNPs.

Here, $W_{\mathrm{G}}=1587.63$.

Applying all these values in equation (1):

$$
1587.63=1581.6+\frac{11}{1+n^{1.6}} .
$$

Hence, $n \approx 1$.

From this equation, we have found that the GNPs are in a single layer.

Again, the weak D band has $I_{\mathrm{D}} / I_{\mathrm{G}}$ ratio $\approx 0.15$ and $I_{2 \mathrm{D}} / I_{\mathrm{G}}$ ratio $\approx 1.921$.

These data are in good agreement with the results obtained from equation (1). So, it is worth pointing out that the GNPs are in a single layer. A defect-free single layer graphene has $I_{2 \mathrm{D}} / I_{\mathrm{G}}$ ratio as 2 , because of lack of $\mathrm{D}$ band or a weak $\mathrm{D}$ band and a sharp symmetric 2D band.

Figure $4 \mathrm{~b}$ shows the Raman spectroscopy of chitosanfunctionalized GNPs. There is slight increase in the $I_{\mathrm{D}} / I_{\mathrm{G}}$ intensity ratio from 0.15 to 0.23 that reveals the functionalization of GNPs through reaction of amine group of chitosan with carboxyl group of GNPs. This indicates a relatively high level of defects in GNPs intensity ratio of $D$ and $G$ bands that depicts the density of defects. Again, this ratio shows no significant change in the structure of GNPs functionalization. The peak at $1587 \mathrm{~cm}^{-1}$ is related to $\mathrm{NH}_{2}$ group of chitosan that proves the functionalization of GNPs.

Figure 5a reveals the Raman spectra of UPE/ESOA/ $0.3 \mathrm{wt} \%$ nanofiller filled nanocomposite. Here, the intensity of D, G and 2D bands of GNPs are very low. This may be due to the low concentration of reinforcement phase of GNPs in the nanocomposite. Figure $5 \mathrm{~b}$ displays the Raman spectra of UPE/ESOA/0.5 wt\% CS/GNPs nanocomposite. Here, D peak is observed due to the dispersion of CS/GNPs nanofiller in the blend and the Raman bands of the UPE/ESOA blend observed were almost negligible. This D band gives the information regarding the amount of impurities in the nanocomposite, which helps in the study of crystallinity. The $G$ and 2D bands of the CS/GNPS nanofiller in the blend were clearly observed approximately at lower wavenumber. This shifting may be attributed to the successful interaction with the formed strong interfacial bonding and hydrogen bonding between the nanofiller and the blend. Figure 5c shows the Raman shift of the nanocomposite with $0.7 \mathrm{wt} \%$ of nanofiller content. In this 


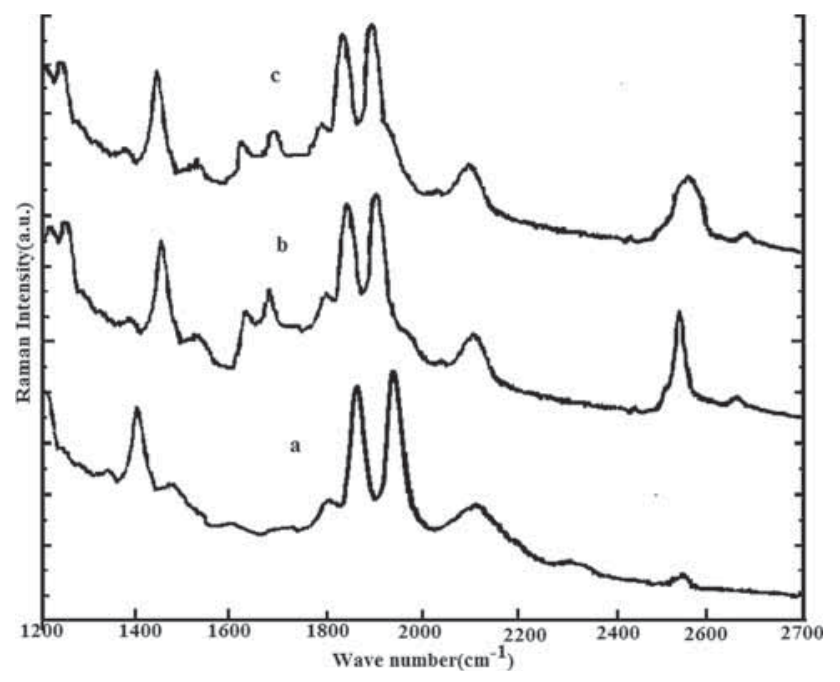

Figure 5. Raman spectra of (a) UPE/ESOA/0.3 wt\% CS/GNPs, (b) UPE/ESOA/0.5 wt\% CS/GNPs and (c) UPE/ESOA/0.7 wt $\%$ CS/GNPs nanocomposites.

case, broad peaks are observed in higher wavenumber for $\mathrm{G}$ and 2D bands of GNPs. Here, a large difference in intensity was observed than in the $0.5 \mathrm{wt} \%$ filled nanocomposite. It may be due to the agglomeration of the nanofiller. We can say that agglomeration of nanoparticles gives higher shift and broadening of the Raman spectrum.

\subsection{SEM}

Figure $6 \mathrm{a}$ reveals the morphological observation of single layer raw GNPs. It can be represented as very smooth and flake-like structure. After dispersion in ethanol, this layer shows the well-dispersed and fully exfoliated GNPs into transparent and individual sheets without any sign of agglomeration, which is shown in figure 6b. The surface morphology of CS/GNPs shows an irregular interface having grooves of polar groups with rigidly smooth morphology. This shows a better chemical bonding between the CS and GNPs. Here, GNPs are covered by a chitosan layer that proved its successful functionalization. In addition, the surface morphology of the nanocomposite with $0.5 \mathrm{wt} \% \mathrm{CS} / \mathrm{GNPs}$ loading exhibit a better homogeneity by indicating the unification of nonbiodegradable GNPs nanofiller and biodegradable chitosan. The enhanced density of this nanocomposite proved the strong interaction between CS/GNPs and UPE/ESOA blend. The nanocomposite with only $0.5 \mathrm{wt} \% \mathrm{r}(\mathrm{G})$ exhibits a regular surface without any chitosan particle on the interface. Finally, $0.7 \mathrm{wt} \%$ of nanofiller concentration (figure 6e) depicts the huge amount of clustered CS/GNPs owing to strong Van der Waals attraction resulting in poor dispersion of the CS/GNPs in the blend. This leads to reduction in the properties of the nanocomposite at this particular concentration.

\subsection{TEM}

The morphology of single layer GNPs, GNPs-ethanol dispersion, CS-functionalized GNPs and the nanocomposite with optimum characteristics were characterized by TEM technique. The maximum dispersion of GNPs in ethanol solution was performed by TEM. The typical TEM image of GNPs before dispersion (figure 7a) indicates the smooth and clean surfaces. After dispersion in ethanol by sonication, the GNPs become highly transparent with less-wrinkled surfaces as represented in figure $7 \mathrm{~b}$. This confirms the successful preservation of original structure of GNPs with smooth surface having no defects. Figure $7 \mathrm{c}$ represents the TEM image of CSgrafting on the GNPs at a temperature of $70^{\circ} \mathrm{C}$. This particular grafting temperature allows the chitosan to wrap the surface of the GNPs completely. So, a better homogeneous, highly dispersed functionalization of chitosan occurred without any aggregation. The observed surface structure of CS/GNPs can be attributed to the amide bond between the carboxyl group of GNPs and amino groups of the CS. Figure 7d shows the TEM image of UPE/ESOA/0.5r $(\mathrm{G})$ nanocomposite. There are some dark features, which can be recognized by the bonding between the polymeric chain and GNPs. Figure 7e indicates the nanoscale dispersion of $0.5 \mathrm{wt} \%$ CS-grafted GNPs in the mixture of UPE/ESOA blend. This shows the uniform distribution of CS-functionalized GNPs on the surface of nanocomposite. This chitosan functionalization causes an important structural change, which attributes to the interaction of epoxide group of GNPs and amino group of chitosan with hydroxyl, carboxyl and carbonyl moieties in the UPE/ESOA matrix by making a very stable nanocomposite. Figure $7 \mathrm{f}$ reveals the agglomeration of nanofillers with weak crosslinking between the reinforced nanofillers and the matrix.

\subsection{Mechanical properties}

According to table 1, the tensile strength and Young's modulus of the nanocomposites with $0.5 \mathrm{wt} \%$ of raw GNPs (designated as $0.5 \mathrm{r}(\mathrm{G})$ ), exhibits lesser values than the other varied contents of CS/GNPs nanocomposites. Even $0.3 \mathrm{wt} \%$ of functionalized GNPs shows around $25 \%$ increment than the $0.5 \mathrm{r}$ (G)-reinforced nanocomposite. This demonstrates the successful reinforcement of CS/GNPs as superior reinforcing filler than the raw GNPs. With increased addition of the functionalized nanofillers into the UPE/ESOA blend, enhanced these properties. The addition of CS/GNPs nanofillers effectively increases the strength of the nanocomposite by acting as a support to resist the maximum part of the load through effective interfacial stress transfer. This increasing tendency is noticeable up to the $0.5 \mathrm{wt} \% \mathrm{CS} / \mathrm{GNPs}$ loading. The above properties depreciate in $0.7 \mathrm{wt} \%$ nanofiller content about $23 \%$ than $0.5 \mathrm{wt} \% \mathrm{CS} / \mathrm{GNPs}$ content due to the formation of large clusters through aggregation of the nanofillers. It happens because of the inhomogeneous dispersion of the nanofillers in the matrix due to the high surface energy [18]. 


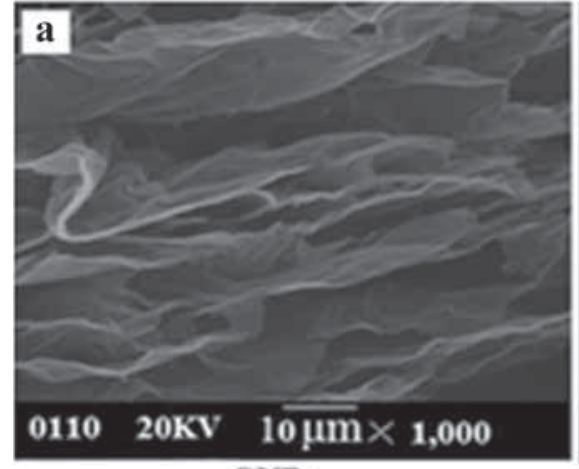

\section{GNPs}

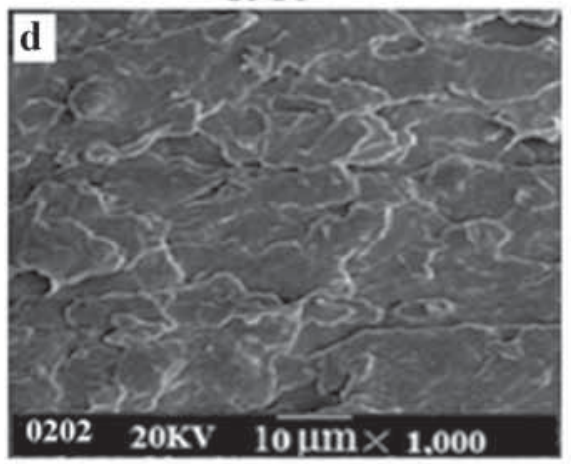

$0.5 \mathrm{r}(\mathrm{G}) / \mathrm{UPE} / \mathrm{ESOA}$

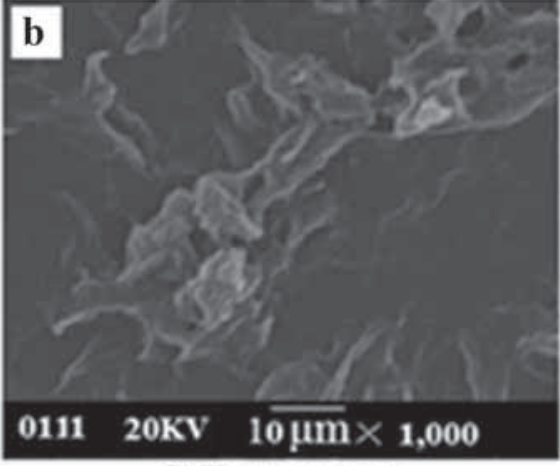

GNPs in ethanol

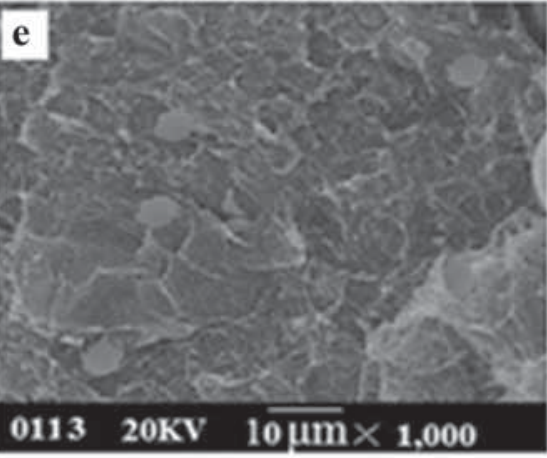

$0.5 w t \% \mathrm{CS} / \mathrm{GNPs}$ nanocomposites

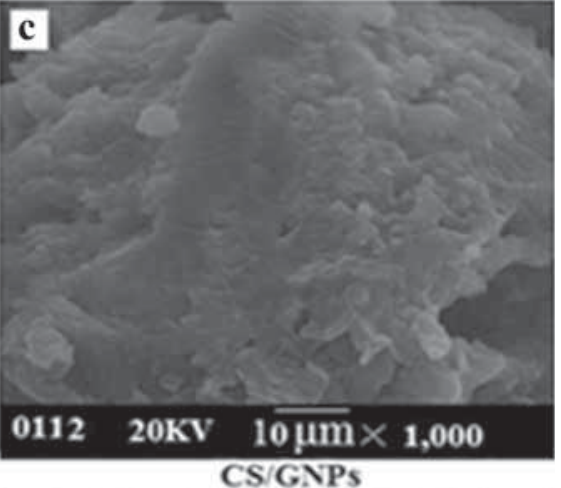

\section{f}

$012020 \mathrm{KV} \quad 10 \mu \mathrm{m} \times 1,000$

$0.7 \mathrm{wt} \% \mathrm{CS} / \mathrm{GNPs}$ nanocomposites

Figure 6. SEM of (a) GNPs, (b) GNPs in ethanol, (c) CS/GNPs, (d) 0.5r (G)/UPE/ESOA, (e) 0.5 wt\% CS/GNPs nanocomposite and (f) $0.7 \mathrm{wt} \% \mathrm{CS} / \mathrm{GNPs}$ nanocomposite.
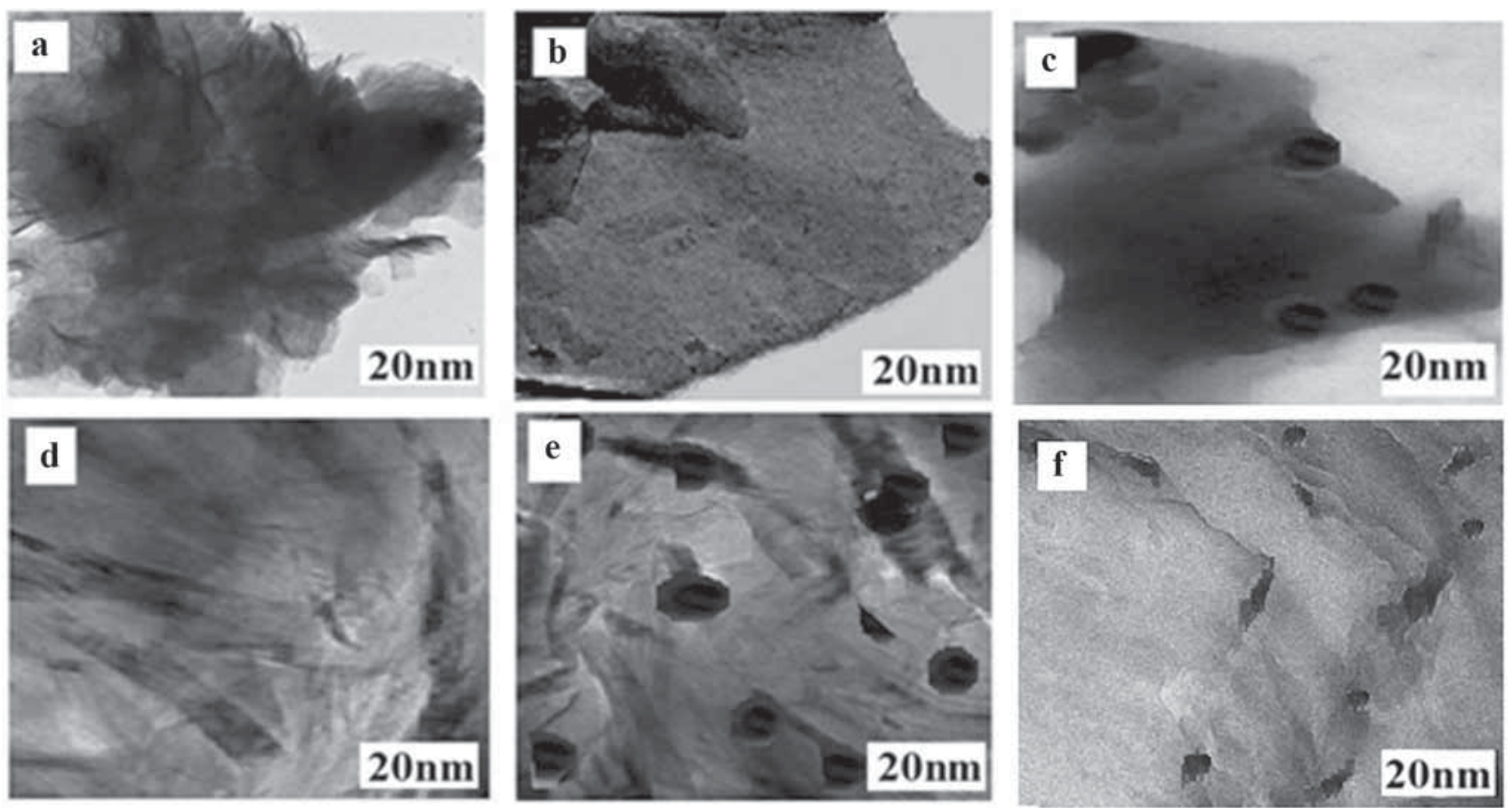

Figure 7. TEM of (a) GNPs, (b) GNPs in ethanol, (c) CS/GNPs, (d) $0.5 \mathrm{r}$ (G)/UPE/ESOA, (e) 0.5 wt $\%$ CS/GNPs nanocomposite and (f) $0.7 \mathrm{wt} \% \mathrm{CS} / \mathrm{GNPs}$ nanocomposite. 
Table 1. Mechanical properties of different types of nanocomposites.

\begin{tabular}{lccccc}
\hline Samples & Tensile strength (MPa) & Strain (\%) & Young's modulus (GPa) & Impact strength (J m $\left.{ }^{-1}\right)$ & Hardness (shore D) \\
\hline 0.5 r G & $56 \pm 3$ & $2.0 \pm 0.2$ & $3.84 \pm 0.1$ & $65 \pm 3$ & $49 \pm 3$ \\
0.3 CS/GNPs & $69 \pm 4$ & $1.8 \pm 0.3$ & $4.45 \pm 0.2$ & $89 \pm 2$ & $57 \pm 1$ \\
0.5 CS/GNPs & $94 \pm 3$ & $1.9 \pm 0.2$ & $5.27 \pm 0.1$ & $115 \pm 4$ & $82 \pm 2$ \\
0.7 CS/GNPs & $73 \pm 3$ & $1.7 \pm 0.2$ & $4.15 \pm 0.1$ & $98 \pm 4$ & $69 \pm 3$ \\
\hline
\end{tabular}
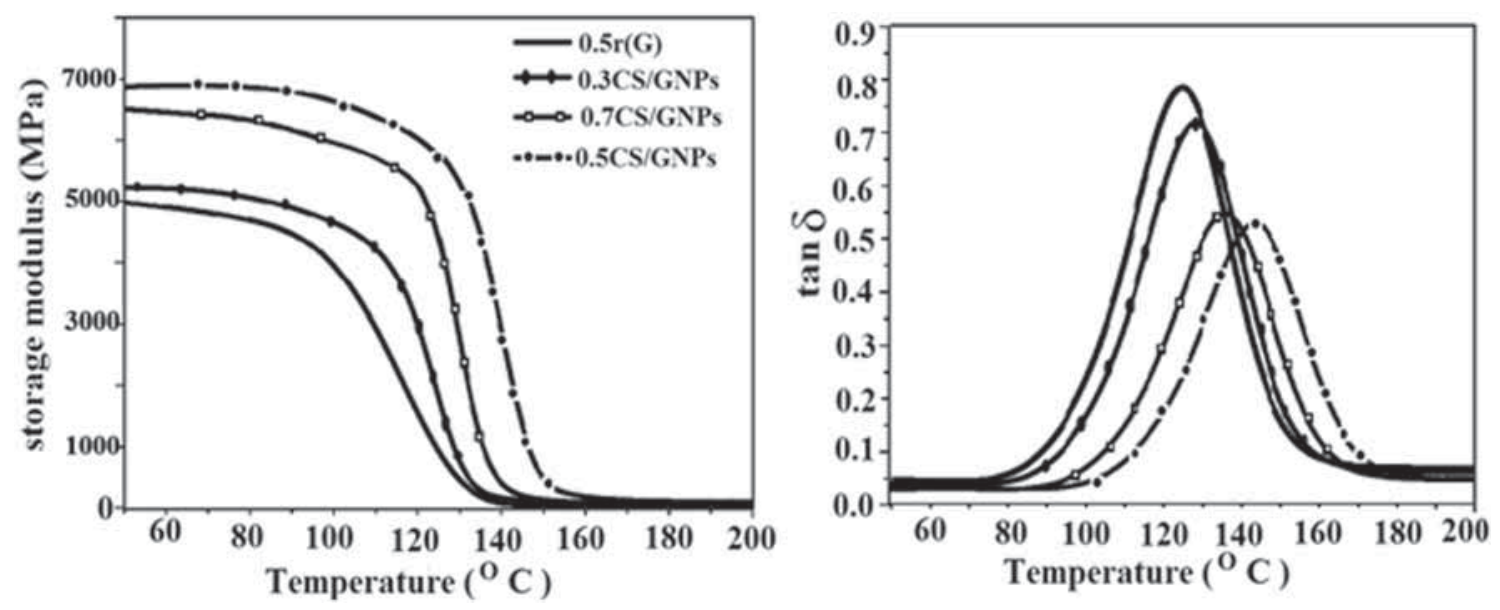

Figure 8. DMA study of different types of nanocomposites.

Meanwhile, the results of impact strength and hardness given in table 1 suggest a regular increment with the incorporation of $0.5 \mathrm{r}(\mathrm{G})$ and other varied contents of CS/GNPs into the UPE/ESOA blend. Here also, the CS-functionalized GNPs proved its dominancy over the raw GNPs. It is due to the effective grafting of chitosan on the GNPs by making it very efficient reinforcing filler. Hence, its proportion was enhanced from 0.3 to $0.7 \mathrm{wt} \%$ according to the effect of the properties on the nanocomposites. It was observed that impact strength and hardness attained a maximum value of 115 and $82 \mathrm{~J} \mathrm{~m}^{-1}$, respectively, at $0.5 \mathrm{wt} \%$ nanofiller content. It may be due to the better matrix-filler interaction, which helps the easy dispersion of nanofillers. But the value deteriorates at $0.7 \mathrm{wt} \%$ nanofillers, because of the enhanced brittleness of the nanocomposites. This makes the restricted movement of the polymer chains by enhancing the crystallinity. So, when impact occurs, this results in micro-cracks on the surface.

For this reason, the higher agglomeration of CS/GNPs nanofillers can cause the decline in mechanical properties of the composites [19].

\subsection{Dynamic mechanical analysis}

Generally, DMA is used to measure two different modules of the nanocomposites, the storage modulus $\left(E^{\prime}\right)$, which is correlated to the capability of the material to store the mechanical energy and loss modulus $(\tan \delta)$ associated with the structural performance of the nanocomposites based upon their glass transition temperature $\left(T_{\mathrm{g}}\right)$. Figure 8 represents the significant enhancement in the storage modulus as well as $T_{\mathrm{g}}$ of the nanocomposites with varied amounts of nanofiller content. The increment in the properties of the CS-functionalized GNP nanocomposites in comparison with the UPE/ESOA blend, and $0.5 \mathrm{r}(\mathrm{G})$ may be due to the better interfacial bonding and enhanced rigidity due to grafting of chitosan. At $0.5 \mathrm{wt} \%$ of the CS/GNPs loading, the storage modulus gives maximum value with reduced damping $(\tan \delta)$ peak, because of the lesser molecular motion of polymer chain due to the strongest cross-linking between filler and matrix, requirement of more molecular energy to move the polymer molecules and lack of interfacial voids. At $0.7 \mathrm{wt} \%$ of nanofiller loading, the aggregation of nanofillers, results in free space and encourage free movement of polymeric molecules due to the low energy absorption. Therefore, it shows low storage modulus with enhanced damping coefficient. Again the $\tan \delta$ values of the nanocomposites are shifted to higher temperature than the $0.5 \mathrm{wt} \% \mathrm{r}(\mathrm{G})$ with rising glass transition temperature. This confirms the higher crystallinity of the nanomaterial with better dispersion and capacity to take up highest vibration. 

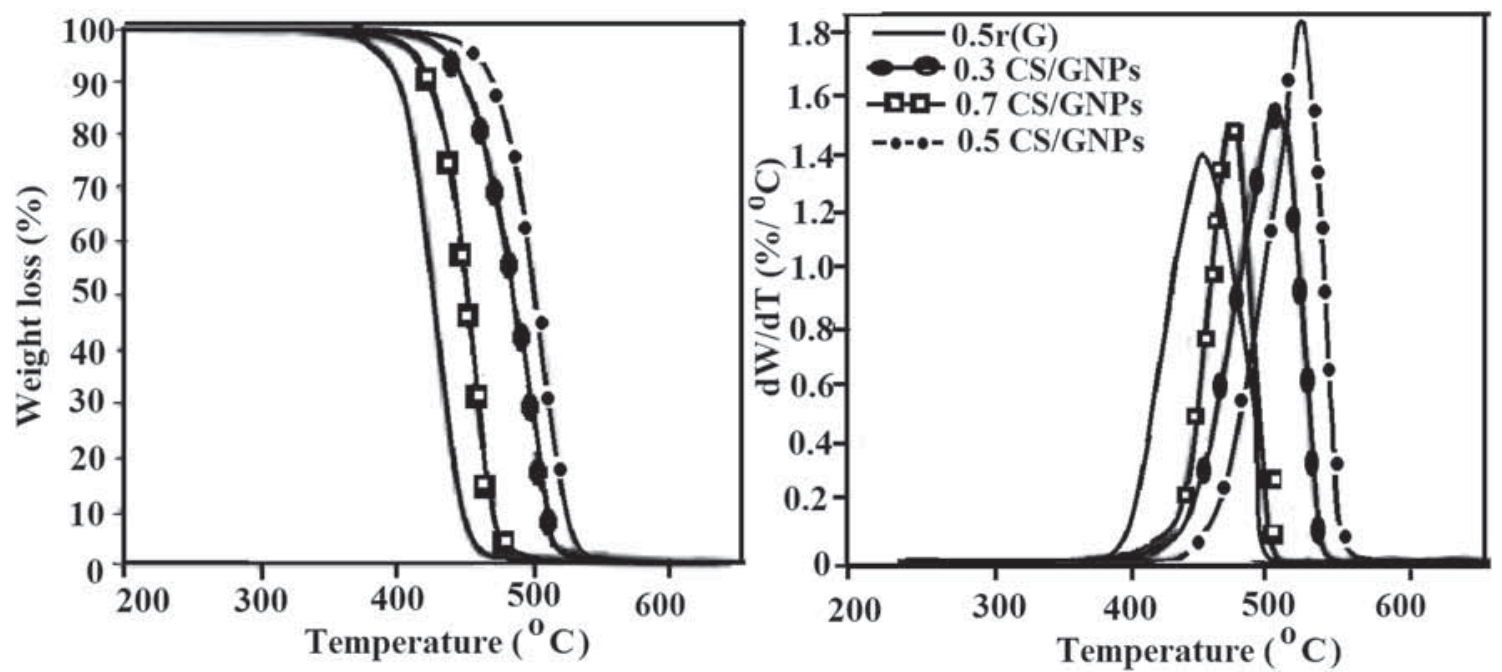

Figure 9. TGA of different types of nanocomposites.

\section{$4.8 T G A$}

Figure 9 depicts the thermal decomposition of the nanocomposites reinforced with $0.5 \mathrm{r}(\mathrm{G})$ and varied contents of CS/GNPs nanofillers. The thermogram indicates only one phase of decomposition for all the nanocomposites. The weight loss under $150^{\circ} \mathrm{C}$ was due to the loss of water molecules [20] and beyond $150^{\circ} \mathrm{C}$ is referred to the loss of bonded water molecules present in the nanocomposite polymeric chain. For the CS-GNPs nanofiller-reinforced nanocomposites, the degradation occurs at higher temperature range than $0.5 \mathrm{r}(\mathrm{G})$-filled nanocomposite. The initial degradation temperature of $0.5 \mathrm{CS} / \mathrm{GNPs}$ nanofiller-reinforced composites showed about $23 \%$ higher temperature compared to the $0.5 \mathrm{r}(\mathrm{G})$-filled nanocomposites, signifying an enhanced thermal stability. This increased thermal stability may be due to the better cross-linking and compatibility between the blend and the CS-grafted GNPs nanofillers. The presence of these nanofillers with optimum concentration of $0.5 \mathrm{wt} \%$ results in the compactly packed structure without any cracks and voids and induces thermal resistance. In addition, the $T_{\max }$ shows higher value confirming higher thermal stability at this particular concentration. But incorporation of $0.7 \mathrm{wt} \% \mathrm{CS} / \mathrm{GNPs}$ nanofillers into the blend matrix showed about $10 \%$ reduced thermal decomposition temperature than its corresponding $0.5 \mathrm{wt} \% \mathrm{CS} / \mathrm{GNPs}$ filled nanocomposite due to aggregation of fillers and poor surface characteristics resulting in thermal instability.

\subsection{Thermal conductivity}

Figure 10 represents the nanofiller-concentration dependence of thermal conductivity of the UPE/ESOA blend-based composites containing $0.5 \mathrm{r}(\mathrm{G}) \mathrm{CS} / \mathrm{GNPs}$ nanofillers with various concentrations. The thermal conductivity value enhances regularly with the increase in the nanofiller loading.

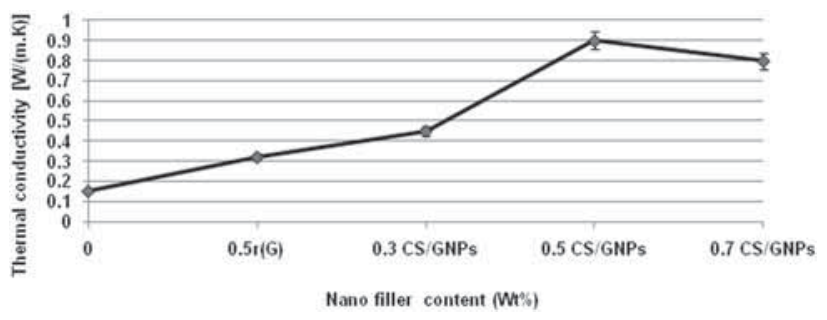

Figure 10. Thermal conductivity of UPE/ESOA blend and different nanocomposites.

0.3 wt $\%$ CS/GNPs-filled nanocomposite showed better thermal conductivity than $0.5 \mathrm{r}(\mathrm{G})$. Moreover, at this concentration, the nanofiller amount is so small that it cannot disperse on the surfaces and damps the vibrational amplitude of the phonon and reduces the conductivity value. It may be due to the formation of a strongly bonded interface due to successful formation of bonds between CS/GNPs and the blend. This value increases up to $0.5 \mathrm{wt} \%$ of CS/GNPs loading, because of better dispersion of the nanofiller, which ease the movement of phonons form GNPs to the matrix system. At this particular concentration of nanofiller, highest thermal conductivity is observed especially due to the homogenous dispersion of GNPs that makes easier to create heat conductive paths. But when thermal conductivity decreases at a concentration of $0.7 \mathrm{wt} \% \mathrm{CS} / \mathrm{GNPs}$ due to the aggregation of nanofillers, it results in very weak cross-linking between the reinforcednanofiller and the matrix.

\subsection{Electrical properties measurement}

4.10a Dielectric strength: The dielectric strength of the nanocomposites is indicated in figure 11. The nanocomposites reinforced with $0.5 \mathrm{r}(\mathrm{G})$ showed increased dielectric strength 


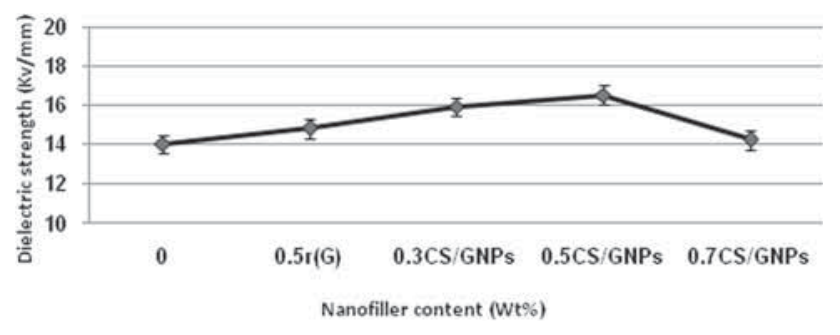

Figure 11. Dielectric strength of UPE/ESOA blend and different nanocomposites.

compared to the raw UPE/ESOA blend. This may be due to the intrinsic electrical properties, quasi-dimensional and platelet structure of the GNPs [21]. Now, the nanocomposites reinforced with changeable amount of CS/GNPs nanofiller were studied. The nanocomposites with $0.5 \mathrm{wt} \% \mathrm{CS} / \mathrm{GNPs}$ nanofiller showed optimum dielectric strength due to the enhanced total surface area offered from the nanofiller as well as its stability and continuity. Also, improved bonding stuck between the matrix and the chitosan-functionalized GNPs resulted in higher dielectric strength [22]. On the other hand, the dielectric strength decreases in $0.7 \mathrm{wt} \% \mathrm{CS} / \mathrm{GNPs}$ content due to the agglomeration of the nanoparticles that reduces the proper dispersion of the nanofiller by enhancing the viscosity of the mixture, which has a very negative effect on overall properties of the nanocomposites.

$4.10 \mathrm{~b}$ Electrical conductivity: Figure 12 depicts the volume and surface conductivities of UPE/ESOA nanocomposites with $0.5 \mathrm{wt} \%$ of raw GNPs as well as varying contents of CS/GNPs nanofillers. Both the conductivities improved up to $0.5 \mathrm{wt} \%$ of CS/GNPs nanofiller and decreased thereafter. On incorporation of $0.5 \mathrm{wt} \% \mathrm{r}(\mathrm{G})$, the electrical conductivity of the nanocomposites enhanced quickly. But the utmost volume conductivity of $6.5 \mathrm{E} \pm 10^{-5} \mathrm{~S} \mathrm{~cm}^{-1}$ and surface conductivity of $3.8 \mathrm{E} \pm 10^{-7} \mathrm{~S} \mathrm{~cm}^{-1}$ were resulted in the nanocomposites containing $0.5 \mathrm{wt} \%$ of CS/GNPs nanofiller may be due to the better conductive network formed inside the composite. The consequences displayed the excellent uniform dispersion and large specific surface area of the nanofiller, which created a good conducting network in the insulating resin matrix. Here, the ensuing conductivity is greater than that of G-reinforced UPE resin matrices [23].

\subsection{Corrosion testing}

In figure 13, the histogram represents the weight gain (oxidation) of the nanocomposites on exposure to $10 \mathrm{wt} \% \mathrm{NaCl}$ solution with changing content of various nanofiller loadings. Inspite of increased hours of $\mathrm{NaCl}$ treatment, very small weight was gained in all the nanocomposites. However, the weight gain is negligible in the nanocomposites with

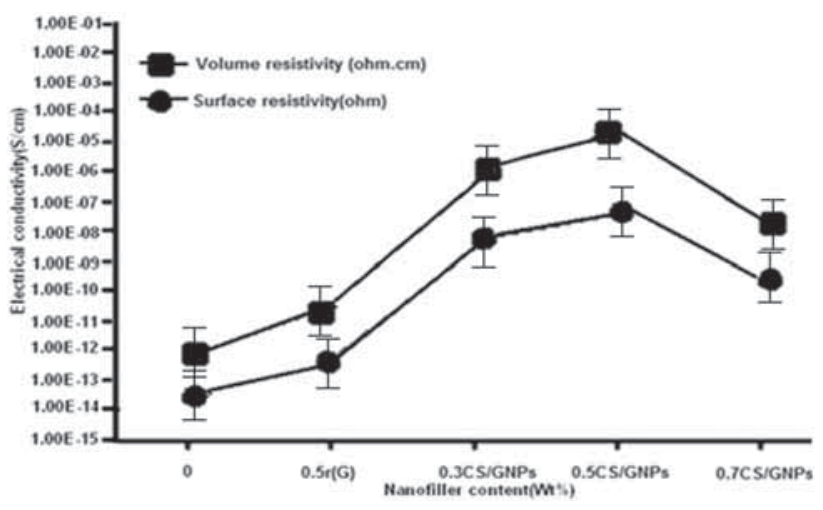

Figure 12. Electrical conductivity of UPE/ESOA blend and different nanocomposites.

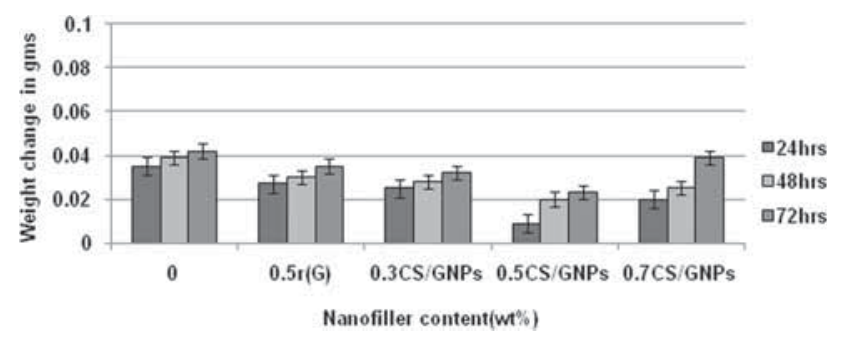

Figure 13. Corrosive properties of UPE/ESOA blend and different types of nanocomposites.

$0.5 \mathrm{wt} \% \mathrm{CS} / \mathrm{GNPs}$ nanofiller as compared to the $0.5 \mathrm{r}(\mathrm{G})$, owing to the stronger interfacial bonding between the matrix and chitosan-grafted nanofiller. This confirms the successful grafting mechanism of chitosan on the GNPs nanofiller. Here, CS/GNPs reduce the chances of formation of voids in the composite system due to the proper homogeneous distribution of the reinforcing filler. So, the diffusion rate of salt water inside the matrix is decreased and diminished weight change is observed at $0.5 \mathrm{wt} \%$ content of CS/GNPs. On the other hand, due to the agglomeration of nanofiller in the case of $0.7 \mathrm{wt} \%$ of CS/GNPs loading, micro voids are formed in the matrix. This leads to the diffusion of salt water inside the nanocomposite by showing an enhanced weight gain [24].

\subsection{Swelling properties}

A structured glimpse of data for the swelling property of nanocomposite to find out their usefulness in a variety of solvent-based application is illustrated in figure 14. This shows a highly significant and prominent swelling behaviour through changes in weight after exposing to different solvents. All the nanocomposites are less vulnerable to the solvents compared to the pristine UPE/ESOA resin. This demonstrates highly cross-linked systems. But the nanocomposite with $0.5 \mathrm{wt} \%$ of GNPs exhibited lowest swelling trend than other systems owing to improved adhesion with the blend matrix and hydrogen bonding between the nanofiller and matrix. 


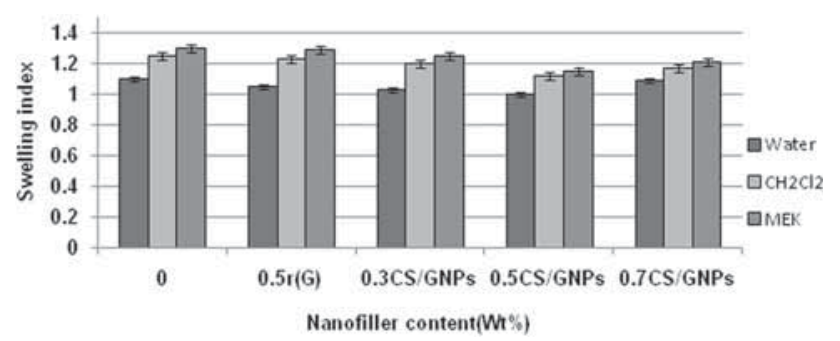

Figure 14. Swelling properties of UPE/ESOA blend and different types of nanocomposites.

But with increase in the nanofiller content up to $0.7 \mathrm{wt} \%$ of CS/GNPs, the solvent ingress increased due to the agglomeration, cracks and micro voids on the nanocomposite surface causing debonding between matrix and filler particle [25]. Hence, the nanocomposites with $0.5 \mathrm{wt} \%$ of CS/GNPs can exhibit superior stability and high applicability for various solvent-related applications as well as for other structural applications due to better mechanical and thermal properties.

\section{Conclusion}

The distinctiveness of this work presents a valuable and unique method of chemically attached biodegradable chitosan on the graphene nanoplatelets as nanofiller with varying proportions for the first time into UPE/ESOA (80:20) blend matrix. The overall outcome showed from FTIR, SEM and TEM that they have compatibility with possibility of coreaction. The resulting materials have established much attention due to their potential to gain properties superior to traditional engineering materials. The studies discovered major improvements in morphological, mechanical, dynamic mechanical, thermal and electrical properties along with reduced corrosion and swelling properties that have a great deal of application in engineering and nanoelectronics fields. The nanocomposite showed pre-eminent properties at $0.5 \%$ CS/GNPs concentration, which is better than the normal $0.5 \mathrm{r}$ (G)-reinforced nanocomposite. Among all the nanocomposites of various concentrations, the material with $0.5 \mathrm{CS} / \mathrm{GNPs}$ nanofillers proved it to be the best one due to the enhanced confinement effect of the nanofiller with the blend. At higher concentrations of $0.7 \mathrm{CS} / \mathrm{GNPs}$ nanofillers, content-free space availability between the filler and matrix increases. This tendency positively affects the growth of microcracks, which hinders the curing process and limits the whole performances of the nanocomposite. Finally, for the above nanocomposite, we can envisage that the novel CS/GNPs nanofiller of optimum concentration will release avenues for next generation GNPs application in the realm of functionalized partially biomaterials.

\section{Acknowledgements}

The authors of this article are thankful to KIIT University for their support and help. The assistance provided by NIT, Rourkela and IIT, Kharagpur, during the completion of the experimental works are greatly acknowledged.

\section{References}

[1] Potts J R, Dreyer D R, Bielawski C W and Ruoff R S $2011 \mathrm{~J}$. Polym. 552

[2] Stankovich S, Dikin D A, Dommett G H B, Kohlhaas K M, Zimney E J and Stach E A 2006 Nature 442282

[3] Bortz D R, Heras E G and Gullon I M 2012 Macromolecules 45 238

[4] Zaman I, Kuan H C, Meng Q S, Michelmore A, Kawashima N, Pitt T et al 2012 Adv. Funct. Mater. 222735

[5] Shen X J, Liu Y, Xiao H M, Feng Q P, Yu Z Z and Fu S Y 2012 Compos. Sci. Technol. 721581

[6] McAllister M J, Li J L, Adamson D H, Schniepp H C, Abdala A A and Liu J 2007 Chem. Mater. 184396

[7] Fang T H, Chang W J and Yang J C 2014 Dig. J. Nanomater. Biostruct. 91207

[8] Yang X, Li L, Shang S and Tao X 2010 J. Polym. 513431

[9] Wang X, Xing W Y, Zhang P, Song L, Yang H Y and Hu Y 2012 Compos. Sci. Technol. 72737

[10] Ma J, Meng Q S, Zaman I, Zhu S M, Michelmore A, Kawashima N et al 2014 Compos. Sci. Technol. 9182

[11] Wan Y J, Tang L C, Yan D, Zhao L, Li Y B, Wu L B et al 2013 Compos. Sci. Technol. 8260

[12] Teng C C, Ma C C M, Lu C H, Yang S Y, Lee S H, Hsiao M C et al 2011 Carbon 495107

[13] Noble L, Gray A I, Sadiq L and Uchegbu IF 1999 Int. J. Pharm. 192173

[14] Majeti N V and Kumar R 2000 React. Funct. Polym. 461

[15] Dutta P K, Dutta J and Tripathi V 2004 J. Sci. Ind. Res. 6320

[16] Dholakiya B (ed.) 2012 Handbook of unsaturated polyester for specialty applications (New York: Intech) p 167

[17] Panda S K, Mohanty P, Behera D and Bastia T K 2016 J. Appl. Polym. Sci. Article ID 44345

[18] Landel R F and Nielsen L E 1994 (2nd eds) Mechanical properties of polymers and composites (New York : Marcel Dekker) p 377

[19] Jianfen S, Weishi H, Liping W, Yizhe H and Mingxin Y 2007 Compos. Sci. Technol. 673041

[20] Han D, Yan L, Chen W and Li W 2011 Carbohydr. Polym. 83 653

[21] Swain S 2013 Trans. Electr. Electron. Mater. 1453

[22] Vilcakova J, Saha P and Quadrat O 2002 Eur. Polym. J. 38 2343

[23] Le M T and Huang S C 2015 Mater. J. 85526

[24] Abdul Khalil H P S, Jawaid M and Abu Bakar A 2011 Bioresources 61043

[25] Law T T and Ishak Mohd Z A 2010 J. Appl. Polym. Sci. 120 563 